110

\section{THE HMGB1 RECEPTOR RAGE MEDIATES HYPOXIC ISCHEMIC BRAIN DAMAGE IN NEONATAL MICE}

M. Winerdal ${ }^{1}$, M.E. Winerdal ${ }^{2}$, A. Bierhaus ${ }^{3}$, O. Winqvist ${ }^{2}, U$. Andersson ${ }^{1}$, U. Ådén ${ }^{1}$

${ }^{1}$ Dept Woman and Child Health, ${ }^{2}$ Dept Medicine, Karolinska Insitutet, Stockholm, Sweden, ${ }^{3}$ Dept Medicine and Clinical Chemistry, University of Heidelberg, Heidelberg, Germany

Background and aims: Hypoxic ischemic (HI) brain injuries provoke cellular damage and subsequent inflammation that can lead to secondary brain injury. Recent data suggest that the receptor for advanced glycation end products (RAGE) and its ligand high mobility group box 1 (HMGB1) are powerful mediators of ischemic inflammation and neurodegeneration in the adult brain, whereas its effects in the immature brain has not been studied. The aim of the present study was to investigate whether HMGB1 is present in the neonatal brain after $\mathrm{HI}$ and whether inactivation/deletion of RAGE affects neonatal brain damage.

Method: We induced brain injury in neonatal mice pups in 10-day-old RAGE wild type $(+/+)$ and knockout -/- mice using a modification of the Vannucci method. Briefly, we electrocoagulated the left common carotid artery and subjected the mice pups to hypoxia $\left(10 \% \mathrm{O}_{2}\right)$ for $60 \mathrm{~min}$. The injury was evaluated 2 weeks after $\mathrm{HI}$ using immunohistochemistry and beam walking, a test of balance and coordination.

Results: First we confirmed that HMGB-1 is expressed in the neonatal mouse brain. We then found that immunoreactivity increased in the injured area. In the RAGE -/- mice, the brain damage was reduced by more than $70 \%$ compared with RAGE $+/+$ mice $(p<0.01)$. A better functional outcome after $\mathrm{HI}$ was seen in RAGE -/- mice compared with RAGE $+/+$ mice using the beam walking test $(p<$ 0.001).

Conclusions: There is a major detrimental effect of RAGE receptor activation in neonatal mice and HMGB1-RAGE receptor signaling represents an interesting target for neonatal neuroprotection.
111

\section{IMPACT OF THE CASPASE-2 INHIBITOR TRP601 ON APOPTOTIC SIGNALING IN THE DEVELOPING BRAIN DURING HYPEROXIA}

M. Sifringer ${ }^{1}$, V. Boos ${ }^{2}, C$. Börner ${ }^{2}$, C. von Haefen ${ }^{1}$, S. Endesfelder ${ }^{2}$, I. Bendix ${ }^{3}$, E. Jacotot ${ }^{4}$, M. Keller ${ }^{3}$, U. Felderhoff-Mueser ${ }^{3}$

${ }^{1}$ Anaesthesiology and Intensive Care Medicine, ${ }^{2}$ Neonatology, Charité-Universitätsmedizin Berlin, Berlin, ${ }^{3}$ Pediatrics I/ Neonatology, University Clinic Essen, Essen, Germany, ${ }^{4}$ Reproductive Biology, Imperial College London, London, UK

Background and aims: Experimental studies show that oxygen, which is widely used in neonatal medicine for resuscitation and treatment of pulmonary hypertension, triggers a disruption of the maintenance of intracellular redox homeostasis. This disturbance can lead to oxidative stress and furthermore to neuronal apoptosis in the developing brain. The role of caspase-2 in apoptosis is poorly defined. Many in vitro studies of caspase-2 knockdown in cultured cells have implicated this caspase in cell death in response to different signaling pathways. To elucidate mechanisms of the caspase- 2 inhibitor TRP601 and its mode of functioning in the developing brain in the context of hyperoxia, we investigated its impact on the levels of APAF-1, AIF, cytochrome c, caspase-9 and -3.

Methods: Six-day old rats were exposed together with their mothers to $80 \%$ oxygen in the presence or absence of the caspase-2 inhibitor TRP601 (1 $\mathrm{mg} / \mathrm{kg}$ ) and were sacrificed after 12 or $24 \mathrm{hrs}$ of hyperoxia following treatment. Dissected brains were either examined histologically to visualize degenerating cells or were subjected to protein studies.

Results: Oxygen exposure triggered cell death at 12 to $24 \mathrm{hrs}$, which was attenuated by TRP601 treatment. Our protein studies demonstrated an upregulation of APAF-1. AIF, cytochrome c, caspase- 9 and -3 in the cytosolic fraction of brain homogenates after hyperoxia, which reached control levels following TRP601 treatment.

Conclusion: These findings suggest a protective role for the caspase-2 inhibitor TRP601 in the prevention of neonatal oxygen-induced apoptotic brain damage.

Supported by the European Commission (Sixth Framework Program, contract no LSHMCT-2006-036534). 